\title{
Synthèse des visites d'intercomparaison de l'organisation et des pratiques de radioprotection dans des centrales nucléaires
}

\author{
F. DROUET ${ }^{1}$, G. CORDIER ${ }^{2}$, P. CROÜAIL ${ }^{1}$, B. JEANNIN ${ }^{2}$, C. LEFAURE $^{1}$, \\ L. VAILLANT ${ }^{1}$
}

(Manuscrit reçu le 29 juin 2007, accepté le 10 novembre 2007)

RÉSUMÉ Depuis le début des années 90, EDF a fait des efforts importants en matière d'optimisation de la radioprotection des travailleurs dans les centres nucléaires de production d'électricité (CNPE) ce qui s'est traduit par une forte réduction de la dose individuelle (581 intervenants au dessus de $20 \mathrm{mSv}$ en 1996 ; aucun au dessus de $20 \mathrm{mSv}$ et seulement 17 au dessus de $16 \mathrm{mSv}$ en 2006) et de la dose collective moyenne par tranche (réduction de plus d'un facteur deux en dix ans). EDF souhaite poursuivre ses efforts pour améliorer la radioprotection dans les centrales françaises. Une des voies d'amélioration est l'analyse du retour d'expérience de la pratique des centrales étrangères ayant de très bons résultats dosimétriques ou ayant connu une amélioration récente significative de la dosimétrie. Dans cette perspective, entre 2003 et 2006, le CEPN a organisé pour EDF huit visites d'intercomparaison de l'organisation et des pratiques de radioprotection dans des centrales nucléaires à l'étranger. Lors de chaque visite, une équipe de quatre personnes, composée de deux représentants d'EDF et de deux représentants du CEPN, se rend sur place pendant une semaine et rencontre des représentants de la plupart des services impliqués dans la mise en œuvre des thèmes retenus. Plusieurs bonnes pratiques de radioprotection ont été mises en avant lors de ces visites : une bonne intégration de la radioprotection dans le management de la centrale; les efforts de réduction du terme source; l'impact fondamental de la réduction des volumes de travail exposé par l'optimisation des programmes de maintenance et par des modifications entraînant une réduction de la nécessité de maintenance ; une radioprotection de proximité expérimentée ; de nouveaux outils au service de la radioprotection du $21^{\mathrm{e}}$ siècle, en particulier la télésurveillance et la télédosimétrie.

ABSTRACT Synthesis of benchmarking visits on the organisation and practical implementation of radiation protection in nuclear power plants.

Since the beginning of the 90s, EDF has made great efforts to improve ALARA for occupational exposures in the French nuclear power plants. This resulted in the important reduction of the individual doses $(581$ workers received more than $20 \mathrm{mSv}$ in 1996; in 2006 no one received more than $20 \mathrm{mSv}$ and only 17 received more than $16 \mathrm{mSv}$ ) and of the average collective dose per reactor (reduction by more than a factor two in ten years). EDF wishes to continue its efforts. One of the ways of improvement is the analysis of the feedback experience in the foreign power plants

1 CEPN, 28 rue de la Redoute, 92260 Fontenay-aux-Roses, France.

2 EDF/DPN, État Major Radioprotection, 1 place Pleyel, 93282 Saint-Denis Cedex, France. 


\begin{abstract}
having good dosimetric results or with a recent significant improvement of the dosimetric results. Thus, between 2003 and 2006, the CEPN has organized for EDF eight benchmarking visits on the organisation and practical implementation of radiation protection in foreign nuclear power plants. For each visit, a team composed of two representatives from EDF and two representatives from CEPN, goes to the site during a week and meets representatives of services implied in the implementation of the specific topic, which was selected. Several good practices of radiation protection were identified during these visits: the good integration of radiation protection in the management of the plant; the efforts in reducing the source term; the fundamental impact of the reduction of exposed volumes of work through the optimisation of the maintenance programmes and modification of the design reducing the necessity of maintenance; a close and experienced radiological protection; new tools in the service of radiological protection for the 21st century, in particular the use of remote monitoring systems.
\end{abstract}

Keywords: radiation protection / benchmarking / nuclear power plant

\title{
1. Introduction
}

Depuis le début des années 90, une très forte réduction des doses individuelles et collectives a été observée dans les centres nucléaires de production d'électricité (CNPE) d'EDF. Ainsi, en 1996, 581 intervenants avaient reçu une dose supérieure à $20 \mathrm{mSv}$; en 2006, personne n'a atteint la limite de $20 \mathrm{mSv}$ et seulement 17 intervenants ont reçu une dose supérieure à $16 \mathrm{mSv}$. Par ailleurs, en 2006, la dose collective annuelle moyenne par réacteur n'était plus que de 0,69 homme.Sv tandis qu'elle était de 1,96 homme.Sv en 1996. Cette amélioration est en partie due à la systématisation de l'application du principe d'optimisation de la radioprotection (principe $\mathrm{ALARA}^{3}$ ) dans les CNPE. Une comparaison des résultats dosimétriques des réacteurs français avec certains des réacteurs étrangers de même conception montre que des améliorations sont encore possibles. Une des voies d'amélioration est donc l'analyse du retour d'expérience (et notamment des bonnes pratiques en matière de radioprotection) de centrales nucléaires étrangères. C'est pourquoi, depuis 2003, le CEPN organise pour EDF des visites d'intercomparaison de l'organisation et des pratiques de radioprotection dans des centrales nucléaires à l'étranger. C'est en s'appuyant sur les données du réseau $\mathrm{ISOE}^{4}$ que les sites ont été choisis en fonction de leurs excellents résultats dosimétriques ou d'une amélioration nette et récente de ces derniers. Huit visites ont été organisées dans les centrales suivantes :

- Almaraz (Espagne - $2 \mathrm{REP}^{5}$ Westinghouse de $930 \mathrm{MWe}$ ) visitée en juillet 2003 ;

As Low As Reasonably Achievable : aussi bas que raisonnablement possible.

Information System on Occupational Exposure - http://www.isoe-network.net. Le CEPN est centre technique européen de ce réseau et gestionnaire de la base de données internationale.

5 Réacteur à eau pressurisée. 
- Doel (Belgique - 2 REP ACECOWEN ${ }^{6}$ de 400 MWe, 1 REP ACECOWEN de $1000 \mathrm{MWe}^{2} 1$ REP FRAMACECO ${ }^{7}$ de $1000 \mathrm{MWe}$ ) visitée en octobre 2003 ;

- Ringhals (Suède - 1 REB ABBATOM de 830 MWe, 1 REP Westinghouse de 875 MWe et 2 REP Westinghouse de 915 MWe) visitée en octobre 2003 ;

- Sizewell B (Royaume-Uni - 1 REP PCC ${ }^{8}$ de $1200 \mathrm{MWe}$ ) visitée en septembre 2004 (Croüail et al., 2004) ;

- Saint Lucie (États-Unis - 2 REP Combustion Engineering de $840 \mathrm{MWe}$ ) visitée en septembre 2005 ;

- Beznau (Suisse - 2 REP Westinghouse de 365 MWe) visitée en juillet 2006 ;

- Vogtle (États-Unis - 2 REP Westinghouse de $1150 \mathrm{MWe}$ ) et Calvert Cliffs (États-Unis - 2 REP Combustion Engineering de $840 \mathrm{MWe}$ ) visitées en octobre 2006: missions centrées sur les systèmes de télédosimétrie et télésurveillance.

Un questionnaire standard a été établi pour ces visites. Ce questionnaire couvre les domaines de la radioprotection elle-même (organisation, préparation des chantiers, assistance et contrôle, etc.), de la chimie du circuit primaire, de la maintenance, du planning, de la formation des intervenants (y compris des prestataires des entreprises extérieures) et de l'analyse des facteurs explicatifs de l'évolution des expositions.

Lors de chaque visite, une équipe de 4 personnes, composée de 2 représentants d'EDF et de 2 représentants du CEPN, se rend sur place pendant une semaine et rencontre des représentants de la plupart des services impliqués dans la mise en œuvre des thèmes retenus. En général, une visite de la zone contrôlée et des installations de formation est organisée par les hôtes. Le choix des participants EDF a toujours permis d'associer au minimum un représentant de CNPE, sélectionné, sans privilégier sa position hiérarchique, en fonction de ses liens avec la thématique principale retenue pour la visite. Dans tous les cas, il s'agissait pour ces personnes de mettre en perspective leurs pratiques et celles de leurs collègues de la centrale hôte.

Cet article a pour objectif de synthétiser les résultats de ces différentes visites afin de mettre en avant les points clés pouvant expliquer les bons résultats dosimétriques des centrales visitées. Ces points sont illustrés par des exemples issus des visites. Il est important de noter que le fait qu'une centrale ne soit pas citée à caractère d'exemple sur un thème donné ne doit pas être considéré comme la mise en évidence d'une faiblesse de la centrale dans ce domaine précis.

\footnotetext{
6 Association ACEC, Cockerill et Westinghouse Nuclear Europe.

Framatome-ACEC-Cockerill.

8 PWR Power Projects.
} 


\section{La radioprotection bien intégrée dans le management de la centrale}

Le management joue un rôle fondamental dans la motivation des intervenants et par conséquent dans l'efficacité de la mise en œuvre de la radioprotection sur un site. La reconnaissance de l'importance de la radioprotection par la direction et l'affichage clair de ce choix permettent un portage plus facile des exigences sur le terrain par le département en charge de la radioprotection et une meilleure appropriation de ces exigences par les autres spécialités et les prestataires.

\subsection{La radioprotection joue un rôle important au sein de la centrale}

Sur tous les sites visités, la radioprotection occupe une place importante dans les organisations hiérarchiques et fonctionnelles des centrales nucléaires. Ainsi, les responsables du «département radioprotection » occupent souvent un poste managérial très élevé (c'est notamment le cas à Almaraz, Saint Lucie, et Beznau). Par ailleurs, la radioprotection est souvent regroupée avec d'autres fonctions (principalement chimie, logistique nucléaire et/ou environnement) dans un seul et même département (centrales d'Almaraz, Doel, Ringhals, Sizewell B, Beznau). Par contre, elle est la plupart du temps totalement séparée de la sécurité (les deux seules exceptions sont les centrales de Doel et de Ringhals). Enfin, il est important de noter que contrairement à ce qui se fait généralement à EDF, aucune des centrales ne fait la distinction entre les responsabilités hiérarchiques et fonctionnelles en matière de radioprotection ${ }^{9}$.

À Almaraz, le chef du département de la protection radiologique et de l'environnement joue le rôle de bras droit du directeur de la centrale sans en avoir officiellement le titre. Il est membre du comité de direction ${ }^{10}$ de la centrale et participe de façon statutaire aux réunions de coordination de la direction générale de l'exploitant.

À Saint Lucie, suite au constat de la dérive des résultats dosimétriques sur quelques années, la direction du site a placé la radioprotection comme un élément prioritaire, garant du bon fonctionnement et des bons résultats de la centrale. Cela s'est notamment traduit par une augmentation du budget du département radioprotection, une augmentation du nombre de contrôles sur le respect des procédures par les intervenants et un effort sur le programme de formation des

\footnotetext{
9 En effet, à EDF, on distingue la radioprotection opérationnelle qui est exercée par le service compétent et l'animation, qui, comme elle concerne l'ensemble des corps de métiers, est faite par le cadre de direction en charge de la radioprotection. Ce cadre de direction n'a aucune fonction hiérarchique sur le service radioprotection.

${ }^{10}$ Ceci est général à l'Espagne du fait que l'autorité de sûreté et de radioprotection l'exige.
} 
intervenants en radioprotection. Il est important de noter qu'aux États-Unis en général, l'INPO ${ }^{11}$ considère que la radioprotection est un élément clé de l'intercomparaison entre les sites. D'ailleurs, l'indicateur de dose collective est utilisé, avec d'autres indicateurs INPO, pour la détermination du montant des primes d'assurance des centrales ${ }^{12}$.

À Beznau, le chef du département radioprotection, chimie et déchets radioactifs, est aussi directeur adjoint de la centrale. À Doel, les radioprotectionnistes ont le pouvoir d'arrêter un chantier. Ainsi, le département radioprotection a déjà annulé - ou reporté - des chantiers de modifications principalement du fait de «dérives » dosimétriques ${ }^{13}$ par rapport aux prévisionnels.

\subsection{La fixation d'objectifs et le suivi régulier d'indicateurs de performance pour la radioprotection}

Des objectifs en matière de radioprotection sont systématiquement fixés par la direction des sites. Outre des critères de doses (collective et individuelle, annuelles et par arrêt de tranche), il existe aussi des critères sur la propreté radiologique, la contamination, les incidents, les «presque incidents », etc. Cependant, compte tenu de la diversité de ces derniers critères et des personnes qui les fixent (exploitant, autorité, etc.), une intercomparaison est plus difficile qu'avec les critères dosimétriques.

Ces objectifs sont le plus souvent fixés annuellement et s'inscrivent parfois dans le cadre de l'établissement d'objectifs pluriannuels (Almaraz, Doel, Saint Lucie). Dans certaines centrales, ces objectifs sont évalués et analysés tous les 6 mois (Doel, Ringhals). En général, l'atteinte ou non de ces objectifs en matière de radioprotection entre dans les critères de choix du montant de la prime annuelle attribuée aux cadres et parfois même de celle qui est attribuée à l'ensemble des employés du site (Doel, Ringhals). Parallèlement, des indicateurs de performance (relatifs à la radioprotection mais aussi parfois à la sécurité, à l'environnement, etc.) sont régulièrement suivis (le plus souvent tous les mois) par le département en charge de la radioprotection et transmis à la direction du site. Le tableau I indique le nombre d'indicateurs de performance qui sont suivis dans les différentes centrales.

${ }^{11}$ Institute of Nuclear Power Operation - organisation créée par l'industrie nucléaire américaine pour promouvoir les plus hauts niveaux de sûreté et de fiabilité dans les centrales nucléaires. Pour cela, l'INPO émet des recommandations pour aider les centrales à mettre en œuvre et maintenir de bonnes pratiques en radioprotection. Tous les opérateurs de centrales nucléaires aux États-Unis sont membres de l'INPO.

${ }^{12}$ Le montant de la prime d'assurance sera d'autant plus faible que la dose collective du site est faible.

13 Par exemple, une dose collective élevée atteinte trop rapidement selon les radioprotectionnistes. 
TABLEAU I

Nombre d'indicateurs de performance suivis par le département en charge de la radioprotection dans les différentes centrales.

Number of performance indicators followed by the radiation protection department in the different nuclear power plants.

\begin{tabular}{cc}
\hline Installation & Nombre d'indicateurs de performance \\
\hline Almaraz & 9 \\
Doel & 9 \\
Sizewell B & Plusieurs dizaines \\
Saint Lucie & 17 \\
Beznau & 13 \\
Calvert Cliffs & 16 \\
Vogtle & 14 \\
\hline
\end{tabular}

\subsection{Une politique ALARA clairement affichée}

Dans la majorité des centrales, il existe un «programme ALARA ${ }^{14}$ » (Almaraz, Doel, Ringhals, Saint Lucie, Vogtle, Calvert Cliffs) permettant de formaliser la démarche ALARA lors des travaux, principalement de maintenance, et un comité ALARA (Almaraz, Doel, Saint Lucie, Beznau, Vogtle, Calvert Cliffs), notamment en charge de la revue des études ALARA relatives aux travaux à fort enjeu dosimétrique. Ces organisations ALARA sont en place et fonctionnent depuis plus de dix ans. Il faut noter que les prestataires ne font jamais formellement partie des comités ALARA. Cependant, il existe des échanges permanents avec les prestataires sous forme de dialogues directs ou de boîtes à idées qui alimentent les réflexions de ce comité. Enfin, compte tenu de la place de la radioprotection dans certaines centrales (poste managérial élevé), les arbitrages en faveur de la radioprotection se font presque systématiquement au niveau de la direction.

En termes d'organisation, les choix retenus sont différents d'un site à l'autre : ils reflètent un contexte et des contraintes propres à chaque site. Ainsi, à Almaraz, des travaux sur les générateurs de vapeur, fort coûteux sur le plan dosimétrique, ont conduit à la création d'une ingénierie radioprotection ad hoc et le lancement d'un programme spécifique de réduction des doses (Programme REDOS) dès 1984, qui a ensuite été accepté en tant que programme ALARA par l'autorité espagnole. Par ailleurs, la direction de la centrale a mis en place un comité ALARA et plusieurs groupes de travail ALARA thématiques : groupe de préparation du travail, groupe ALARA, groupe d'amélioration ALARA. Même si

${ }^{14}$ Un programme ALARA définit précisément les instances décisionnelles, les procédures et les responsabilités en matière d'optimisation de la radioprotection dans une centrale. 
aucun prestataire ne fait partie de ces groupes, ces derniers participent systématiquement aux prévisionnels dosimétriques lors de la préparation des « chantiers ALARA ».

À Doel, c'est la mise en place très tôt d'une contrainte de dose ${ }^{15}$ qui a été à l'origine d'une réflexion particulière sur l'organisation des ressources humaines et qui a abouti à la mise en place d'un programme ALARA renforcé.

Beznau a fait le choix de créer en 1998 une équipe ALARA, pluridisciplinaire, composée de 9 personnes issues de 4 départements différents et dirigée par le chef du service radioprotection. Ce groupe se réunit régulièrement (35 réunions depuis 1998) afin de préparer des plans d'action de radioprotection pour la mise en place de moyens d'optimisation, de participer à la préparation des arrêts de tranche (optimisation des travaux les plus dosants) et de suivre la coopération internationale en radioprotection, notamment via la participation au réseau $\mathrm{VGB}^{16}$. Là non plus, les prestataires ne participent pas à ce groupe. Ils ne participent pas plus aux démarches ALARA pour la préparation des chantiers.

\section{Actions sur les débits de dose}

La maîtrise du terme source et des débits de dose associés est un des éléments déterminants pour limiter la dosimétrie des travailleurs. Ce terme source, issu de la contamination des circuits suite à l'activation sous flux de neutrons des produits de corrosion, peut être réduit par des actions sur les matériaux utilisés ou sur la chimie du primaire permettant de contrôler le phénomène de corrosion. D'autres actions comme la pose de protections biologiques ou la propreté radiologique visent à se protéger de ce terme source.

\subsection{Agir sur le design de la centrale}

Plusieurs sites ont travaillé sur l'optimisation du design de leurs installations, que ce soit dès la conception (agencement optimisé grâce à un effort d'analyse détaillée du retour d'expérience d'autres centrales à Sizewell $\mathrm{B}^{17}$ ) ou à l'occasion des opérations de maintenance ou de changement des équipements afin de réduire les termes sources.

$15 \mathrm{La}$ contrainte de dose individuelle annuelle était de $15 \mathrm{mSv}$ lorsque la limite de dose réglementaire était de $50 \mathrm{mSv} / \mathrm{an}$; elle est aujourd'hui de $10 \mathrm{mSv}$.

16 VGB PowerTech e.V. (http://www.vgb.org/startpage.html) - association technique allemande regroupant des producteurs d'énergie. Dans le cadre du comité sur les centrales nucléaires, un groupe de travail radioprotection, auquel participe des représentants de la centrale de Beznau, est mis en place.

${ }^{17}$ La centrale de Sizewell a divergé pour la première fois en 1995 
De façon systématique, que ce soit en France ou à l'étranger, les nouveaux générateurs de vapeur installés contiennent moins de nickel et de cobalt que les précédents, permettant ainsi de réduire l'activation et donc le débit de dose aux alentours.

À Almaraz, durant la dernière décade, les deux tranches ont bénéficié d'efforts considérables sur le design pour favoriser la réduction du terme source. Le remplacement des générateurs de vapeur et des couvercles de cuve, et la suppression des by-pass RTD ont été réalisés en 1996 sur la tranche 1 et en 1997 sur la tranche 2. Des blindages des lignes de purge de la cuve du réacteur ont également été installés lors de ces deux arrêts de tranche. Un programme de réduction des stellites a été mis en place et était toujours en cours en 2004 : il n'y a pratiquement plus aucun robinet stellité. Les matériaux structurels des assemblages combustibles (grille, squelette) sont à basse teneur en cobalt depuis 1996-97.

\subsection{Une chimie du circuit primaire étroitement liée à la radioprotection}

Sur la majorité des sites visités (Doel, Ringhals, Sizewell B, Saint Lucie, Beznau), beaucoup d'efforts portent sur la gestion de la chimie du circuit primaire en fonctionnement ou en arrêt, permettant ainsi de réduire le terme source. Ces efforts portent, entre autres, sur :

- l'optimisation du taux d'acidité (Ringhals, Beznau);

- l'optimisation de la durée d'oxygénation au début de l'arrêt (Ringhals);

- l'addition de zinc (Saint Lucie, essais à Vogtle et Calvert Cliffs) ;

- l'optimisation du traitement d'épuration d'eau (Saint Lucie).

Parallèlement, les centrales ont mis en place une collaboration étroite entre les radioprotectionnistes et les chimistes ce qui va, à Beznau et à Sizewell B (et même à Ringhals avant réorganisation), jusqu'à réunir ces deux spécialités dans un même département.

\subsection{Une optimisation de la pose des protections biologiques}

À Doel et à Beznau, l'optimisation de la pose des protections biologiques en arrêt de tranche afin de faire de cette opération une tâche planifiée à part entière, organisée et optimisée, est présentée comme un facteur explicatif des bons résultats dosimétriques. Les deux objectifs principaux sont de développer une réelle ingénierie de la pose des protections biologiques afin d'optimiser la quantité et les lieux d'implantation, et de professionnaliser l'installation de ces protections. 
À Doel, la société responsable de la pose des protections biologiques a préparé depuis plusieurs années un programme standard d'installation de ces protections en début d'arrêt de tranche. Environ 40 tonnes de plomb sont posées à chaque arrêt. Il existe notamment des points d'ancrage permanents ce qui permet d'optimiser le temps de pose. Les intervenants de cette société affectés à la pose de ces protections sont très qualifiés et entraînés. Ils sont les seuls intervenants du site avec les radioprotectionnistes (prestataires et du site) à être habilités à réaliser des mesures de débit de dose.

À Beznau, le service radioprotection, en charge du programme des protections biologiques, a développé une nouvelle politique au début des années 2000 afin de réduire la quantité de protections utilisées en arrêt. Les protections biologiques en plomb sont posées avant le début des travaux de maintenance uniquement dans les zones où il y a des travaux durant l'arrêt. Cette politique a permis de réduire de moitié la quantité de plomb (environ 80 tonnes de plomb par arrêt avant 2000, environ 40 tonnes après 2000) utilisé sans pénaliser les résultats dosimétriques en arrêt.

\subsection{Un effort important pour la propreté radiologique et la surveillance des locaux}

Les centrales nucléaires sont des installations nécessitant un programme de maintenance très important avec également une forte co-activité. Dans ce contexte, le maintien de la propreté radiologique des locaux est un élément majeur pour éviter toute contamination lors des opérations mais aussi à l'issue des opérations, pour rendre au poste de travail son aspect originel en prévision des prochains travaux.

Le site de Beznau est extrêmement propre. Toute contamination est systématiquement nettoyée ou surveillée le cas échéant. L'opérateur a le devoir de nettoyer son poste de travail à l'issue de son activité. Parallèlement, une entreprise spécialisée effectue en permanence un nettoyage du sol dans la zone contrôlée. Une des conséquences de cette propreté est qu'aucune contamination interne n'a été détectée depuis environ 30 ans. Concernant la surveillance, pour chaque tranche, le service radioprotection réalise des mesures (contamination ou irradiation) sur environ 70 points quotidiennement et sur environ 70 autres points chaque semaine

À Sizewell B, lorsqu'un chantier est terminé, l'opérateur radioprotection doit vérifier la contamination de tous les outils utilisés dans la zone de repli. 


\section{Actions sur les volumes de travail exposé}

La réduction des volumes de maintenance (par un travail approfondi d'optimisation des opérations de maintenance ou par des modifications entraînant une réduction de la nécessité de maintenance) a une incidence évidente sur les volumes de travail exposé et par conséquent sur la dosimétrie. Plusieurs sites (Almaraz, Doel, Ringhals, Beznau) affichent une réelle volonté d'optimiser le volume horaire des travaux de maintenance lors des arrêts de tranches afin d'éviter les travaux inutiles, participant ainsi à la réduction des expositions. Par exemple, plusieurs centrales ont fortement réduit, en accord avec les autorités de sûreté, la fréquence du contrôle de leurs générateurs de vapeur.

À Almaraz, suite aux remplacements des générateurs de vapeur, l'état des tranches permet de ne contrôler qu'un seul des trois générateurs de vapeur à chaque arrêt. Le volume de travail de maintenance est d'ailleurs globalement en baisse du fait de la propreté radiologique des deux tranches.

À Doel, depuis une dizaine d'années, des efforts sont systématiquement faits pour réduire les temps de travail, notamment en réduisant le nombre de contrôles jugés inutiles (par exemple en ne faisant aucune maintenance préventive sur les matériels et organes qui ne sont pas classés «importants pour la sûreté ou la production »). L'autorité de sûreté belge a également accepté, après négociations avec l'exploitant, que les deux générateurs de vapeur ne soient contrôlés qu'une fois tous les 6 ans (au lieu du contrôle d'un générateur de vapeur chaque année). La cadence de contrôle choisie par l'exploitant ${ }^{18}$ permet ainsi deux arrêts de tranche consécutifs sans ouverture de générateur de vapeur. Finalement, le volume de travail pour les opérations de maintenance est inférieur à 30000 personnes.h pour une visite principale (supérieur à 40000 personnes.h pour les réacteurs 900 MWe en France), et inférieur à 50000 personnes.h pour une visite décennale (supérieur à 90000 hommes.h pour les réacteurs 900 MWe en France).

À Ringhals, le volume des interventions de maintenance, décidé principalement par la centrale elle-même sous la surveillance des autorités de sûreté, est plus faible qu'en France. L'exploitant cherche à raccourcir la durée des arrêts de tranche : 29 jours en 2004, 22 jours en 2005, 17 jours en 2006. En France, pour les $900 \mathrm{MWe}$, la durée des arrêts est systématiquement supérieure à 30 jours, même lors des arrêts pour simple rechargement ${ }^{19}$.

\footnotetext{
18 Ouverture des deux générateurs de vapeur lors du même arrêt tous les trois arrêts.

${ }^{19}$ Généralement plus de 50 jours pour les VP et plus de 100 jours pour les VD.
} 


\section{Une radioprotection de proximité expérimentée}

\subsection{Une collaboration étroite entre les radioprotectionnistes et les autres métiers}

Sur tous les sites, les radioprotectionnistes travaillent en relation directe avec les autres métiers, notamment pour la préparation et la réalisation des arrêts de tranche. Cela se traduit par une présence forte des radioprotectionnistes sur le terrain au plus près des intervenants et des chantiers. Ils sont notamment très présents en zone contrôlée pour le contrôle et le conseil et il existe systématiquement un bureau radioprotection en entrée de zone, point de passage obligatoire pour la délivrance du permis de travail radiologique.

À Doel, la collaboration entre les préparateurs radioprotection et les préparateurs métiers a été renforcée depuis quelques années. La réorganisation du site a permis d'impliquer encore plus ces derniers dans les phases de préparation de leurs chantiers sur le plan de la radioprotection, dans les prévisions dosimétriques et dans les analyses d'écarts. Enfin, la préparation du travail s'est considérablement améliorée (disponibilité des outillages et pièces de rechange, formation des intervenants à leur utilisation).

À Ringhals, les radioprotectionnistes sont en contact direct avec les travailleurs pendant toute la durée de l'arrêt (bureau de la radioprotection, radioprotectionnistes présents en permanence dans l'enceinte de confinement, etc.).

À Sizewell B, une approche assez pragmatique de la radioprotection a été adoptée depuis le démarrage de la centrale. Par de nombreux aspects, la façon de travailler est apparue moins procédurale que dans d'autres centrales, mais en raison de l'existence d'un dialogue important et permanent entre les radioprotectionnistes et les autres métiers (et notamment les prestataires en arrêt de tranche), elle a encouragé d'autres groupes de professionnels, tels que ceux affectés à la maintenance de l'installation, à parler très ouvertement et à travailler avec le personnel de radioprotection. Il faut noter que les ingénieurs et techniciens radioprotection possèdent une grande expérience du site, une connaissance détaillée des travaux réalisés par les autres groupes et de nombreux techniciens radioprotection ont une formation en matière d'ingénierie mécanique qui les aide à comprendre les tâches exécutées par les autres groupes.

\subsection{La gestion des compétences et la formation en radioprotection}

Le turn-over, notamment dans les départements radioprotection, est généralement très faible dans les centrales visitées. La plupart des employés ont de nombreuses 
années d'expérience sur le site, certains étant même présent depuis la première divergence (Almaraz, Sizewell B). Cela n'a pas pour origine que l'arrivée de jeunes spécialistes en radioprotection est insuffisante pour assurer le renouvellement. Cela implique par contre que le personnel connaît parfaitement la centrale, son organisation et son état radiologique.

À Almaraz, la plupart des employés (cadres et techniciens) sont là depuis les premières divergences des tranches. Les moins expérimentés ont une quinzaine d'années d'expérience. À Doel, le turn-over est quasiment nul en ce qui concerne les techniciens en radioprotection. Au moins la moitié du personnel a plus de 10 ans d'expérience du site. À Sizewell B, la plupart des agents des équipes de radioprotection travaillent dans la centrale depuis la première divergence. On peut ainsi dire que l'équipe radioprotection représente la «mémoire » de l'installation.

À Saint Lucie, même si le turn-over n'est pas inexistant, les nouveaux techniciens radioprotection arrivant sur le site ont déjà une assez grande expérience acquise sur d'autres sites, notamment à Turkey Point. Par ailleurs, les prestataires ont aussi majoritairement beaucoup d'ancienneté et sont en plus bien intégrés dans l'équipe radioprotection. Si historiquement, aux États-Unis, les radioprotectionnistes étaient majoritairement issus de la marine, ce n'est plus le cas et une réflexion est en cours afin de créer de nouvelles filières de formation en radioprotection.

En ce qui concerne la formation en radioprotection des intervenants non radioprotectionnistes, la durée et les thèmes abordés dépendent de la catégorie de personnel. Cette formation est à la fois théorique et pratique. Pour les employés permanents, une remise à niveau est réalisée tous les ans (Almaraz, Doel, Saint Lucie) ou tous les deux ans (Sizewell B, Beznau). Ces formations sont parfois sanctionnées par un examen (Doel, Ringhals, Saint Lucie, Beznau). Pour les prestataires, la remise à niveau est annuelle et est systématiquement sanctionnée par un examen. Enfin, à Sizewell B, à Saint Lucie et à Calvert Cliffs, des chantiers écoles sont organisés permettant ainsi aux intervenants de se former sur des maquettes en zone non irradiante.

\section{De nouveaux outils au service de la radioprotection du $21^{\mathrm{e}}$ siècle}

\subsection{Le système d'information adapté à la radioprotection}

Une centrale nucléaire est une installation extrêmement complexe et en constante évolution. Un grand nombre de données, et notamment de données radiologiques, sont nécessaires à la maintenance de cette installation. Dans la plupart des 
centrales visitées, un effort d'organisation de ces données a été effectué. L'accès à certaines de ces données n'est pas réservé aux seuls radioprotectionnistes mais est autorisé pour tous les préparateurs des autres spécialités voire pour les intervenants eux-mêmes.

Certaines centrales disposent de bases de données informatiques et photographiques facilement consultables regroupant diverses informations et paramètres (niveaux de contamination, débits de dose, relevés historiques de la dosimétrie et des paramètres chimiques du circuit primaire, etc.).

À Almaraz, le département en charge de la radioprotection dispose d'une base de données cartographiques très complète des conditions radiologiques des deux tranches : 300 cartographies de locaux sont informatisées, contenant pour chaque zone l'historique des valeurs de 3 à 10 points de mesure de débit de dose d'ambiance, ou au contact des matériels - et ce, en fonction des différents états de la tranche avant ou pendant les arrêts de tranche. La base de données contient également les mesures de contamination atmosphérique (aérosols et halogènes) et surfacique. Les intervenants peuvent la consulter depuis le local radioprotection situé à l'entrée de la zone contrôlée. Les cartographies sont remises à jour régulièrement et la plus récente est affichée à l'entrée de chaque local.

La centrale de Doel dispose d'un logiciel nommé SARA contenant une base de données des plans de l'installation. Tous les gros matériels (vannes importantes, pompes, échangeurs, etc.) y sont repérés. Chaque zone peut contenir les informations suivantes: contamination surfacique et volumique, débit de dose ambiant, débit de dose au contact, activité, concentration en iode, débit de dose neutron, présence d'alpha, commentaires. Des mesures sont disponibles depuis 1990.

À Sizewell B, les radioprotectionnistes établissent des fiches d'informations, comprenant des photographies, des plans d'implantation et des informations sur les débits de dose de rayonnements, pour de nombreux composants de la centrale. Les intervenants ont également la possibilité de connaître leur zone de travail à l'aide d'un logiciel nommé Surrogate-Tour.

La centrale de Beznau a développé un logiciel informatique regroupant les mesures quotidiennes de contamination et d'irradiation ${ }^{20}$ et leurs évolutions. Ces paramètres peuvent être consultés depuis les bureaux radioprotection situés en entrée de zone contrôlée et en zone contrôlée. Un autre programme informatique (CIS - Chemistry Information System) permet de suivre les différents paramètres

${ }^{20}$ Voir paragraphe 3.4 . 
chimiques des circuits primaires et secondaires en temps réel. Les données sont conservées dans une base et sont consultables à tout moment.

\subsection{Apports des technologies de télésurveillance et télédosimétrie}

\subsubsection{Généralités}

Un système de suivi à distance des données (Remote Monitoring System - RMS) est généralement constitué de trois éléments distincts :

- un système de télédosimétrie permettant la transmission et la centralisation en temps réel des informations fournies par un ensemble de dosimètres électroniques équipés de télétransmetteurs (jusqu'à plusieurs centaines de dosimètres) ;

- un système de télésurveillance qui permet de suivre visuellement, voire d'enregistrer, le déroulement d'un travail ;

- un système de communication audio permettant à l'ensemble des personnels (travailleurs exposés, techniciens radioprotection de terrain et techniciens radioprotection en charge du suivi à distance) de communiquer (d'individu à individu ou à un groupe d'individus).

Le système (tout ou en partie) peut s'appuyer sur un transfert de données sans fil. L'utilisation de technologies sans fil - émettrices d'ondes radio - nécessite de réaliser une cartographie des zones sensibles aux longueurs d'ondes utilisées pour le transfert de données afin de ne pas interférer avec l'instrumentation en place.

\subsubsection{Intérêt pour la radioprotection}

\section{Diminution des expositions}

Le RMS permet le suivi à distance (depuis la zone non contrôlée pour les centrales de Saint Lucie, Vogtle et Calvert Cliffs), des conditions d'exposition aux rayonnements ionisants des travailleurs: localisation et identification $\mathrm{du}$ travailleur; nature du travail et données relatives au prévisionnel dosimétrique (notamment seuils d'alarme en dose collective, dose individuelle et débit de dose) ; débit de dose ; durée d'exposition ; dose individuelle. La flexibilité du système permet par ailleurs de suivre l'évolution des débits de dose en tout lieu, ce qui tend à favoriser un grand nombre d'applications : suivi du taux d'encrassement de filtres ou suivi du transfert des éléments de combustible par exemple. Par ailleurs, les données (mesures) produites par les balises de suivi de la contamination atmosphérique (aérosol et iode) peuvent être également télétransmises et suivies. Enfin, la centralisation en un lieu unique de l'ensemble de ces informations permet un suivi efficace et réactif des travailleurs exposés par un personnel réduit. Cela 
permet de s'affranchir de la présence permanente des techniciens radioprotection sur le terrain, tout en conservant leur rôle d'accompagnement et de conseil par l'intermédiaire des liaisons phoniques.

À Sizewell B, lors de sa première utilisation en 2004, il a été estimé que le système de surveillance audio et vidéo en réseau et de moyens de dosimétrie en temps réel a permis de réduire d'environ $10 \%$ la dose totale de l'arrêt (c'est-à-dire 20 hommes.mSv), le personnel radioprotection étant le groupe qui a le plus bénéficié de cette réduction.

À Vogtle, le nombre de techniciens radioprotection contractuels en période d'arrêt de tranche est passé de 118 à 40 en quelques années avec la mise en œuvre du RMS. L'exposition radiologique du personnel radioprotection en arrêt de tranche est passée de $0,4^{21}$ à 0,1 homme. $S^{22}$. Aujourd'hui, l'exposition radiologique du personnel RP en arrêt de tranche est d'environ 0,05 homme.Sv.

De 1999 à 2006, à Calvert Cliffs, le nombre de techniciens radioprotection contractuels en période d'arrêt de tranche est passé de 93 à 62 et le département radioprotection a vu son effectif passer de 63 à 44 permanents. L'exposition radiologique du personnel radioprotection est passée de 0,3 (1999) à 0,14 homme.Sv (2006).

Toutefois, on ne s'affranchit pas de la présence des techniciens radioprotection à proximité des personnels exposés. En effet, lorsqu'un technicien du centre de surveillance détecte un écart - accroissement rapide du débit de dose, dépassement du prévisionnel dosimétrique, contamination atmosphérique, etc. -, l'envoi d'un technicien radioprotection sur le terrain est immédiat.

\section{Formation et préparation des chantiers}

En matière de formation et de préparation, l'utilisation de la vidéo (enregistrement de tâches spécifiques) permet de préparer un travail, d'améliorer le geste technique et de prendre connaissance avec l'équipement concerné (en particulier lors du «pre-job briefing »). Par ailleurs, le suivi centralisé des informations permet d'archiver l'ensemble des caractéristiques radiologiques d'un travail et de préparer au mieux les prévisionnels dosimétriques.

\section{Acceptabilité sociale}

En termes d'acceptation sociale, en s'appuyant sur l'exemple de la centrale de Vogtle, il semble qu'une démarche progressive, s'appuyant sur des groupes de

21 Dose collective moyenne avant mise en place du RMS

22 Dose collective moyenne après mise en place du RMS 
travail par métier et accordant une place importante à l'échange soit la plus appropriée.

Une attention particulière doit être accordée à la démarche d'appropriation de l'outil RMS par l'ensemble du département radioprotection et une articulation adéquate doit être trouvée entre le temps passé dans la salle de surveillance et le temps passé sur le terrain. Les techniciens radioprotection des sites de Calvert Cliffs et de Vogtle estiment, pour la plupart, que le RMS apporte une valeur et une technicité ajoutées à leur métier.

L'intérêt pour la technologie RMS ne réside pas que dans son emploi dans le cadre de la radioprotection. De nombreux métiers souhaitent avoir accès à cette technologie (suivi du déroulement d'un chantier, amélioration du geste technique). Ces demandes doivent être prises en compte sans pour autant nuire à l'objectif d'amélioration des performances en radioprotection et mobiliser le personnel radioprotection pour des missions ne relevant pas de son domaine d'activité.

\section{Conclusion}

Les huit visites d'intercomparaison de la radioprotection pratique et opérationnelle dans des centrales nucléaires, organisées par le CEPN entre 2003 et 2006, ont permis de mettre en avant plusieurs bonnes pratiques participant à la réduction de la dosimétrie dans ces centrales :

- la place importante de la radioprotection dans l'organisation de la centrale : positions hiérarchiques élevées pour les responsables de la radioprotection, politique ALARA affichée...;

- la réduction du terme source notamment pour les travaux en arrêt de tranche en agissant sur le design de l'installation, la propreté et la surveillance des locaux, la chimie du circuit primaire et la pose des protections biologiques ;

- la réduction des volumes de travail exposé par l'optimisation des travaux de maintenance, une bonne connaissance de l'installation, des formations régulières, une collaboration étroite entre les radioprotectionnistes et les autres métiers ;

- l'utilisation de la télésurveillance et de la télédosimétrie.

Il convient de souligner que les enseignements tirés de ces visites ont permis à EDF d'orienter fortement certains dossiers :

- les exemples de permis de travail radiologique rapportés de toutes les centrales visitées ont alimenté l'étude des ergonomes qui a précédé la mise en place des régimes de travail radiologique à $\mathrm{EDF}$;

- à la suite des visites aux États-Unis, la pratique américaine des «blind tests » lors de la lecture des systèmes de dosimétrie passive par les laboratoires a été 
appliquée à EDF. Il s'agit de faire traiter par le laboratoire des dosimètres témoins ayant été irradiés dans des conditions bien spécifiques afin de vérifier la validité des résultats de ce laboratoire ;

- les informations recueillies sur les systèmes de télésurveillance et de télédosimétrie lors des différentes visites ont alimenté le groupe de travail mis en place à EDF sur cette thématique.

Par ailleurs, ces visites ont permis aux représentants des CNPE de voir comment d'autres exploitants abordent des problèmes similaires aux leurs.

Ces visites d'intercomparaison vont se poursuivre jusqu'en 2009. Parallèlement de nouvelles visites devraient être également organisées dans certaines des centrales déjà visitées afin d'analyser les évolutions en matière de radioprotection depuis la première visite.

\section{RÉFÉRENCE}

Croüail P., Jeannin B., Lefaure C., Panisset L. (2004) Organisation de la radioprotection à la centrale nucléaire de Sizewell B (Royaume-Uni), Rapport CEPN Nº 290. 